HEP/123-qed

\title{
Effect of the break-up on the fusion and elastic scattering of weakly bound projectiles on ${ }^{64} \mathrm{Zn}$
}

\author{
P. R. S. Gomes ${ }^{1}$, M.D. Rodríguez ${ }^{2}$, G.V. Martí , , I. Padron ${ }^{1}$, L.C. \\ Chamon $^{3}$, J.O. Fernández Niello ${ }^{2,4}$, O.A. Capurro ${ }^{2}$, A.J. Pacheco ${ }^{2}$, J.E.

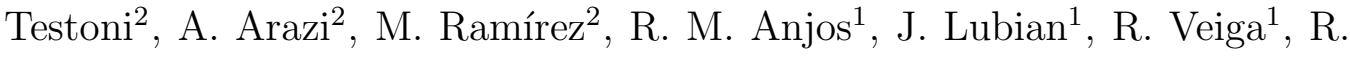 \\ Liguori Neto ${ }^{3}$, E. Crema ${ }^{3}$, N. Added ${ }^{3}$, C. Tenreiro ${ }^{5}$ and M.S. Hussein ${ }^{6}$. \\ ${ }^{1}$ Instituto de Física, Universidade Federal Fluminense, \\ Av. Litoranea s/n, Gragoatá, Niterói, R.J.,24210-340, \\ Brazil. ${ }^{2}$ Laboratorio Tandar, Departamento de Física, \\ Comisión Nacional de Energía Atómica, \\ Av. del Libertador 8250, (1429), Buenos Aires, \\ Argentina. ${ }^{3}$ Departamento de Fúsica Nuclear, Universidade de São Paulo, \\ Caixa Postal 66318, 05315-970, São Paulo, S.P., \\ Brazil. ${ }^{4}$ Escuela de Ciencia e Tecnología, Universidad de San Martín, \\ Argentina. ${ }^{5}$ Facultad de Ingeniería, Universidad de Talca, \\ Curicó, Chile. ${ }^{6}$ Departamento de Física Matemática, \\ Universidade de São Paulo, Caixa Postal 66318, \\ 05315-970, São Paulo, S.P., Brazil
}

(Date textdate; Received textdate; Revised textdate; Accepted textdate; Published textdate)

\begin{abstract}
We study the behavior of the fusion, break-up, reaction and elastic scattering of different projectiles on ${ }^{64} \mathrm{Zn}$, at near and above barrier energies. We present fusion and elastic scattering data with the tightly bound ${ }^{16} \mathrm{O}$ and the stable weakly bound ${ }^{6} \mathrm{Li},{ }^{7} \mathrm{Li}$ and ${ }^{9} \mathrm{Be}$ projectiles. The data were analyzed by coupled channel calculations. The total fusion cross sections for these systems are not affected by the break-up process at energies above the barrier. The elastic (non-capture) break-up cross section is important at energies close and above the Coulomb barrier and increases the reaction cross sections. In addition we also show that the break-up process at near and sub-barrier energies is responsible for the vanishing of the usual threshold anomaly of the optical potential and give rise to a new type of anomaly.
\end{abstract}




\section{INTRODUCTION}

The fusion of weakly bound nuclei, both stable and radioactive, has been the subject of intense theoretical and experimental activities 1, 2, 3, 4, 5, 6, 7, 8, 9, 10, 11, 12, 13, 14, 15, 16, 17, 18, 19, 20, 21, 22, 23, 24, 25, 26, 27, 28, 29, 30, 31, 32, 33, 34, 35, 36, 37, 38, 39, 40, 41, 42, 43, 44, 45, 46, 47, 48]. Since the beginning of the 1990's, theorists have been facing conflicting ideas about whether the fusion of such nuclei, of great importance in nuclear astrophysics, is enhanced or hindered at low energies owing to the strong coupling to the break-up channel 10, 11, 12, 13, 14, 15, 16, 17, 18, 19, 20, 21, 22]. The reason for such divergent conclusions has been the lack of a trust-worthy continuum-coupled channels theory. Even recently, when the Continuum-Discretized Coupled Channel (CDCC) theory has been utilized for the calculation of fusion [21, 22], the results are not fully satisfactory due to the difficulty in calculating the incomplete fusion (the fusion of a piece of the projectile) contribution to the total fusion[21].

One of the motivations in studying the fusion of stable weakly bound nuclei with heavy targets is the considerably high intensity of such beams as compared to radioactive beams. Most of the essential features of the phenomenon, namely the importance of the break-up channel even at low energies, the related significance of incomplete fusion, and the fact that what is normally measured is the total fusion (the sum of complete and incomplete fusion), are present in both cases, albeit less conspicuously in the stable isotope case. The discerning of the sought after complete fusion cross section constitutes the real experimental challenge in the field. The recent publication on the ${ }^{6} \mathrm{He}+{ }^{238} \mathrm{U}$ system [23] is an important step in this direction. Yet, detailed investigation of the fusion of stable weakly bound nuclei is still very important as it allows the study of a wider range of systems and the development of much needed systematics.

The suitable stable nuclei for this kind of study are ${ }^{9} \mathrm{Be},{ }^{6} \mathrm{Li}$ and ${ }^{7} \mathrm{Li}$ which have threshold break-up energies between $1.48 \mathrm{MeV}$ and $2.45 \mathrm{MeV}$. The full understanding of the fusion and break-up processes induced by these beams is an important reference for similar studies involving radioactive proton and neutron rich projectiles [7, 8, 9].

Among the questions on this subject that have to be answered, one finds: What are the values of $\sigma_{E B U}$ (EBU or elastic break-up is the break-up not followed by the capture of any of the break-up fragments by the target) for different energy regimes, target masses 
and threshold break-up energies? Does the break-up affect the fusion cross section or just increase the reaction cross section? If it affects the fusion process, does it enhance or suppress the fusion cross section at different energy regimes and target masses? Is the effect on the complete fusion or total fusion, the last one corresponding to the sum of the normal two-body fusion with the fusion processes following break-up (ICF (incomplete fusion) and CFBU (complete fusion following break-up))?

There are several theoretical aspects to be considered when one wants to study the influence of the break-up process on the fusion cross section. One should include all the mentioned different reaction mechanisms related with the break-up and the relative motion of the fragments and their interactions, the boundary conditions used for the occurrence of complete fusion, such as the distance where the fusion is decided, and definitions of $\mathrm{CF}$ and ICF related to bound and unbound states. Calculations have to be performed by considering bound states-continuum couplings, with or without resonance states, discrete continuum states and continuum-continuum couplings, Coulomb and nuclear excitations have to be considered, as well as their coherent interferences. Up to now, there is no such a complete theoretical study in this field. Different approaches have been used, such as coupled channel calculations (CCC) that do not take into account the break-up process 19], continuum discrete coupled channel calculations (CDCC) 20, 21, 22], semi-classical trajectories, survival probability concept and dynamic polarization potentials 10, 11, 12, 13, 14, 15, 16, 17, 18].

Experimentally, it is important to know if it is possible to separate the $\mathrm{CF}$ and ICF processes. Usually the residues following both processes are very similar or identical, and therefore the measurement of residues using different techniques and/or particle identification devices (i.e., charged particle detectors, time of flight detectors, ionization chamber, $\mathrm{x}$-rays delayed, etc.) is not able to distinguish between them. This is even more dramatic for light systems for which the main evaporation channels include charged particles (protons and alphas). Therefore, most of the available data in the literature correspond to total fusion (TF) cross section, although there are reports of some measurements of CF and ICF separately 24, 25, 26, 27, 28, 29, 30]. The measurement of EBU cross sections requires difficult exclusive experiments [31, 32, 33, 34, 35, 36].

The present paper is organized as follows. In Section 2, we present the experimental details on the low energy induced reactions of ${ }^{16} \mathrm{O}$ (stable, tightly bound), ${ }^{6} \mathrm{Li},{ }^{7} \mathrm{Li}$ and ${ }^{9} \mathrm{Be}$ (stable, weakly bound) projectiles on ${ }^{64} \mathrm{Zn}$. In Section 3 we give the experimental results for 
the fusion and the elastic scattering angular distributions, through which the reaction cross sections are extracted and discussed. In Section 4, the elastic scattering angular distributions are analyzed with the São Paulo Optical Potential (SPOP), which takes into account the effect of the nonlocality within the double folding prescription. A new threshold anomaly directly related to the persistent action of the coupling to the break-up channel, even at below barrier energies, is also discussed in this section. Finally, in Section 5, we present our concluding remarks. A short version of a part of this work has already been published 37].

\section{EXPERIMENTAL DETAILS}

The experiments to measure the elastic scattering for the ${ }^{16} \mathrm{O}+{ }^{64} \mathrm{Zn},{ }^{9} \mathrm{Be}+{ }^{64} \mathrm{Zn}$ and ${ }^{6,7} \mathrm{Li}+{ }^{64} \mathrm{Zn}$ systems were performed at the Pelletron Laboratory of the Universidade de São Paulo (USP), São Paulo, Brazil. The elastic scattering cross sections were measured with a set of nine surface barrier detectors, placed at $40 \mathrm{~cm}$ from the target, with $5^{0}$ angular separations between two adjacent detectors having a resolution of the order of $350 \mathrm{keV}$ at $20 \mathrm{MeV}$. In front of each detector, there was a set of circular collimators and slits for the definition of the solid angles and to avoid slit-scattered particles. The experimental array has been previously described in detail in references [38, 39, 40]. The angle determination was made by reading on a goniometer with a precision of $0.5^{0}$. An additional surface barrier detector used as monitor was placed at $20^{\circ}$, relative to the beam direction, for normalization purposes. For the Li isotopes scattering, the relative solid angles of the detectors were determined by Rutherford scattering of ${ }^{6} \mathrm{Li}$ on the ${ }^{197} \mathrm{Au}$ thin backing layer deposited onto the target. The ${ }^{64} \mathrm{Zn}$ target had a thickness of $60 \mu \mathrm{g} / \mathrm{cm}^{2}$. Three beam energies were used for the ${ }^{6} \mathrm{Li}$ beam, 17, 20 and $22 \mathrm{MeV}$, and two energies for the ${ }^{7} \mathrm{Li}$ beam, 20 and $22 \mathrm{MeV}$, with the angular range $10^{\circ} \leq \theta_{L a b} \leq 150^{\circ}$. The uncertainties in the differential cross section data vary from $1 \%$ to $8 \%$. The measurements with the ${ }^{9} \mathrm{Be}$ and ${ }^{16} \mathrm{O}$ beam have been previoulsly reported [38, 39].

The total fusion cross sections of the ${ }^{6} \mathrm{Li}$ and ${ }^{9} \mathrm{Be}+{ }^{64} \mathrm{Zn}$ systems were measured using the experimental facilities of the TANDAR Laboratory, in Buenos Aires, Argentina. Beams of ${ }^{6} \mathrm{Li}$ at 16, 18, 20, 22 and $24 \mathrm{MeV}$, and ${ }^{9} \mathrm{Be}$ at 20, 22 and $24 \mathrm{MeV}$ were delivered by the 20 UD-tandem accelerator. A metallic ${ }^{64} \mathrm{Zn}$ target, with thickness of $50 \mu \mathrm{g} / \mathrm{cm}^{2}$, was deposited on a $10 \mu \mathrm{g} / \mathrm{cm}^{2}$ carbon backing and used for runs collecting data for both measured 
systems. The fusion cross sections data were obtained using the time of flight method and complement previously reported results obtained by the same method, at higher energies, for the ${ }^{6} \mathrm{Li}+{ }^{64} \mathrm{Zn}$ system [41] and by the gamma ray spectroscopy method for the ${ }^{9} \mathrm{Be}+$ ${ }^{64} \mathrm{Zn}$ system [38]. Further description of the detection system and additional experimental details can be found in Ref. 42. Figure 1 shows a typical bi-parametric energy vs. mass spectrum and the projection of this spectrum on the mass axis, for the ${ }^{9} \mathrm{Be}+{ }^{64} \mathrm{Zn}$ system, measured at $\theta_{L a b}=10^{0}$ and $24 \mathrm{MeV}$ beam energy.

\section{EXPERIMENTAL RESULTS FOR FUSION AND REACTION CROSS SEC- TIONS AND DISCUSSIONS}

Table 1a) shows the results for the fusion and the deduced reaction cross sections obtained for the ${ }^{6} \mathrm{Li},{ }^{7} \mathrm{Li}+{ }^{64} \mathrm{Zn}$ systems, together with previously reported fusion data [41]. In Table 1b) we quoted the results of measured fusion and derived reaction cross sections for the ${ }^{9} \mathrm{Be}+{ }^{64} \mathrm{Zn}$ system from the present work and from Ref. 38, derived reaction cross sections from Ref. 39 for the ${ }^{16} \mathrm{O}+{ }^{64} \mathrm{Zn}$ system and the previously reported fusion cross section for the ${ }^{16} \mathrm{O}+{ }^{64} \mathrm{Zn}$ system[43]. It is worthwhile to notice that the total fusion cross sections obtained for the ${ }^{9} \mathrm{Be}+{ }^{64} \mathrm{Zn}$ system by the gamma ray method [38, 44] and using the TOF technique agree very well, as can be seen in Figure 2.

In order to study the possible influence of the break-up of weakly bound nuclei on the fusion and reaction cross sections, we use ${ }^{16} \mathrm{O}+{ }^{64} \mathrm{Zn}$ as a reference system since it has a negligible break-up cross section. Figure 3 shows that total fusion $\left(\sigma_{T F}\right)$ and reaction cross sections $\left(\sigma_{R}\right)$ are similar for the whole energy range. The dashed line is the result of CCFULL 19] calculations without any couplings, and the full line is the result including the couplings of the first two excited states of the target $\left(2_{1}^{+}\right.$and $2_{2}^{+}$states at $\mathrm{E}^{*}\left(2_{1}^{+}\right)=0.792 \mathrm{MeV}$ and $\mathrm{E}^{*}\left(2_{2}^{+}\right)=1.799 \mathrm{MeV}$, respectively). Almost no difference between the two calculations can be observed, as expected at this energies above the Coulomb barrier. A good fit of the total fusion excitation function is obtained.

Figure 4 shows that for the weakly bound projectile ${ }^{9} \mathrm{Be}, \sigma_{T F}$ and the deduced $\sigma_{R}$ are also very similar for most of the studied energy range, but for energies close to the Coulomb barrier, $\sigma_{T F}$ becomes appreciably smaller than $\sigma_{R}$. Here, $\sigma_{T F}$ corresponds to the sum of the fusion of ${ }^{9} \mathrm{Be},{ }^{8} \mathrm{Be}$ and one alpha fragment with the target. Since the inelastic excitation 
of ${ }^{64} \mathrm{Zn}$ has small cross section [38], and supposing that transfer reaction cross sections are also negligible, we conclude from the unitary constrainty $\sigma_{R} \geq \sigma_{T F}+\sigma_{E B U}$ that the cross section of the ${ }^{9} \mathrm{Be}$ break-up into two alpha particles plus one neutron without any capture (EBU) is significative, when compared with $\sigma_{T F}$, only at energies close to the barrier. The full and dashed lines in Figure 4 correspond to CCFULL calculations for the total fusion, performed at similar way as in Figure 3. The derived $\sigma_{E B U}$ values are also shown in Figure 4. Small error bars are obtained for the two lowest energies, since $\sigma_{E B U}$ was derived by the difference between the large $\sigma_{R}$ value and the relatively small $\sigma_{T F}$ value estimated from the CCFULL calculations, whereas for higher energies, the error bars are very large due to the uncertainties coming out from the difference between two large numbers, since $\sigma_{R} \sim \sigma_{T F}$ at this energy regime. A good fit of the total fusion excitation function is obtained, showing that there is no total fusion suppression or enhancement, compared with predictions from the bare potential of the CCFULL code. Therefore, we conclude that the break-up of ${ }^{9} \mathrm{Be}$ does not affect $\sigma_{T F}$, but rather increases $\sigma_{R}$ at energies close to the barrier. From the fusion data obtained by the gamma ray spectroscopy method it was possible to estimate [37, 38] that the $\sigma_{I C F}$ is less $10 \%$ of the $\sigma_{T F}$, where $\sigma_{I C F}$ corresponds to the fusion of one alpha particle fragment with the target [38, 44].

Figure 5 shows $\sigma_{T F}$ and the derived $\sigma_{R}$ for the ${ }^{6} \mathrm{Li}+{ }^{64} \mathrm{Zn}$ system. The total fusion excitation function is well described by CCFULL calculations, without couplings or including the coupling to the first two excited states of the target, leading to the conclusion that $\sigma_{T F}$ is not affected by the presence of the break-up process. It is worth mention that our $\sigma_{T F}$ data are very similar to those for the ${ }^{6} \mathrm{Li}+{ }^{59} \mathrm{Co}$ system [45], and the later were well described by CDCC calculations [46]. Contrary to the behavior with the ${ }^{9}$ Be projectile, $\sigma_{R}$ for the ${ }^{6} \mathrm{Li}$ are larger than $\sigma_{T F}$ even at the highest energies, and therefore $\sigma_{E B U}$ has significant cross section at this regime.

Figure 6 shows $\sigma_{T F}$ and $\sigma_{R}$ for the ${ }^{7} \mathrm{Li}+{ }^{64} \mathrm{Zn}$ system. As there are no $\sigma_{T F}$ data available at low energies, we did not attempt to perform coupled channel calculations. The full line is the result of CCFULL calculations without any coupling. Although the two energies for which $\sigma_{R}$ were derived are slightly lower than the ones for which $\sigma_{T F}$ are available, one can notice that $\sigma_{R}>\sigma_{T F}$ even at energies above the barrier, similar to the behavior with the ${ }^{6} \mathrm{Li}$ projectile. The derived $\sigma_{E B U}$ for this system are shown in Figure 6, where the $\sigma_{T F}$ value used was obtained from the CCFULL predictions. 
From the behavior of the derived $\sigma_{T F}, \sigma_{R}$ and $\sigma_{E B U}$ for the ${ }^{9} \mathrm{Be},{ }^{6} \mathrm{Li},{ }^{7} \mathrm{Li}+{ }^{64} \mathrm{Zn}$ systems and also using the fact that for the ${ }^{6} \mathrm{He}+{ }^{64} \mathrm{Zn}$ system, $\sigma_{R}>>\sigma_{T F}$ at energies above the barrier [37, 47, 48], we draw a scenario as follows: At energies above the barrier, the weakly bound nuclei ${ }^{6} \mathrm{He}$ and ${ }^{6,7} \mathrm{Li}$ break-up at relatively large distances from the target, and their fragments move in different directions, leading to significant $\sigma_{E B U}$ (or, alternatively, the EBU corresponding to large partial waves has significant cross section). In a different breakup process, the ${ }^{9} \mathrm{Be}$ breaks-up into ${ }^{8} \mathrm{Be}$ and one neutron, the ${ }^{8} \mathrm{Be}$ moves almost in the same direction as the ${ }^{9} \mathrm{Be}$, and only sometime later $\left(\sim 10^{-16} \mathrm{sec}\right)$ the two alpha particles are produced. The whole process for the ${ }^{9}$ Be break-up, particularly with a relatively light target with a not very strong Coulomb potential, reduces the probability of EBU at this regime. At energies close and below the barrier, one expects that $\sigma_{E B U}$ becomes significant for any of the weakly bound projectiles, particularly if the target is heavy and the Coulomb break-up takes place at relatively larger distances.

\section{THE ELASTiC SCATTERING ANALYZED WITH THE SÃO PAULO PO- TENTIAL}

The strong presence of the break-up process at near barrier energies affects the elastic scattering and reaction cross section of weakly bound nuclei in such way that the usual threshold anomaly of the optical potential is no longer present, except from ${ }^{7} \mathrm{Li}$ induced reactions, as it was previoulsly reported 30 , 40, 49, 50, 51, 52, 53, 54]. For the ${ }^{9} \mathrm{Be}+$ ${ }^{64} \mathrm{Zn}$ system, in previous works [38, 44], we followed the usual procedure of obtaining the energy-dependence of the real and imaginary parts of the optical potential at the strong absorption radius, using a Woods-Saxon shape for the real and volume imaginary potentials and a derivative Woods-Saxon shape for the surface imaginary potential. The presence of the break-up channel at these low energies does not allow the vanishing of the imaginary part of the potential as the energy approaches the barrier. Indeed, an increase in the imaginary potential is observed at the lowest energy, as it was also observed by Signorini et al. for the ${ }^{9} \mathrm{Be}+{ }^{209} \mathrm{Bi}$ system[53]. On the other hand, for the tightly bound ${ }^{16} \mathrm{O}+{ }^{64} \mathrm{Zn}$ system the usual threshold anomaly is present: the imaginary potential decreases when the bombarding energy decreases towards the barrier [39].

However, the threshold anomaly was recently observed for the ${ }^{9} \mathrm{Be}+{ }^{208} \mathrm{~Pb}$ system [54], 
showing that the behavior of the elastic scattering of weakly bound nuclei is not yet fully understood. Therefore, we decided to study the threshold anomaly by an alternative approach, that uses a global parameter-free optical potential known as São Paulo potential (SPOP) 55, 56]. Very recently, we have applied the same procedure for the study of the elastic scattering of the ${ }^{9} \mathrm{Be}+{ }^{27} \mathrm{Al}$ system[52]. This potential is based on the Pauli nonlocality involving the exchange of nucleons between projectile and target. Within this model, the nuclear interaction is connected with the folding potential $\mathrm{V}_{F}$ through [55, 56] the following formula:

$$
V_{N}(R, E) \approx V_{F}(R) \exp \left(-4 v^{2} / c^{2}\right),
$$

where $c$ is the speed of light in vacuum and $v$ is the local relative velocity between the two nuclei. In this context, and considering an extensive systematics of nuclear densities, a systematization of the imaginary part of the optical potential was also obtained:

$$
W(R, E)=N_{i} V_{N}(R, E) .
$$

For several systems, elastic scattering angular distributions over wide energy ranges were simultaneously well fitted with an optical potential defined by Eqs. (1) and (2), with $\mathrm{N}_{i}=0.78$ [56].

In order to explain the elastic scattering angular distributions for the ${ }^{9} \mathrm{Be}+{ }^{64} \mathrm{Zn}$ system, we started with the SPOP without any free parameter (and consequently no data fit), corresponding to the use of Equation (1), multiplied by $\mathrm{N}_{R}=1.0$, and Equation (2) with $\mathrm{N}_{i}$ $=0.78$ [56]. The results are shown in Figure 7 by dashed lines. Then, the values of $\mathrm{N}_{R}$ and $\mathrm{N}_{i}$ were considered as free parameters to fit the data. The solid lines in Figure 7 correspond to the best data fits. The results of the energy dependence of the best $\mathrm{N}_{R}$ and $\mathrm{N}_{i}$ values are shown in Figure 8. The derivation of the error bars was done as follows: we defined the maximum acceptable $\chi^{2}$ value as $\chi_{\text {Max }}^{2}=\chi_{\text {Min }}^{2}+\chi_{\text {Min }}^{2} / \mathrm{N}$, where $\mathrm{N}$ is the number of points of the angular distribution; and then we found the range of $\mathrm{N}_{R}$ and $\mathrm{N}_{i}$ corresponding to $\chi^{2}$ smaller or equal to $\chi_{\text {Max }}^{2}$.

From Figure 8 one can observe a dramatic deviation of the energy dependence from the so-called threshold anomaly (TA). The value of $\mathrm{N}_{R}$ is roughly 0.9 down to the barrier, where one sees a significant drop at $\mathrm{E}<\mathrm{V}_{B}$. Thus the coupling to the break-up channel results 
in an overall repulsion. The behavior of $\mathrm{N}_{i}$, also shown in Figure 8, is even more dramatic: the value 0.78 seems reasonable up to energies in the vicinity of the barrier. As the energy is lowered further, a significant increase in $\mathrm{N}_{i}$ is observed. This is in sharp contrast to the threshold anomaly behavior seen in the scattering of tightly bound nuclei where one finds the exact opposite behavior of $\mathrm{N}_{i}$ and $\mathrm{N}_{R}$ at $\mathrm{E}<\mathrm{V}_{B}$. The underlying theoretical tool behind the TA is the dispersion relation that relates the real part of the dynamic polarization potential (DPP) - the Feshbach potential - to an energy integral involving the imaginary part. We did not calculate this potential here, but by fitting the data with a slightly modified SPOP, we are effectively taking into account the DPP.

What we are observing here is a new type of threshold anomaly directly linked to the coupling to the break-up channel. We call this the Break-up Threshold Anomaly (BTA). The repulsive nature of the real part of the break-up DPP has been discussed by several authors [57, 58, 59, 60]. The fact that the threshold for break-up extends far down to low energies in the case of weakly bound nuclei is clearly manifested in the BTA. Being repulsive in nature, the break-up DPP would lead to an increase in the barrier height resulting in a decrease of the complete fusion cross section at sub-barrier energies. This should become more conspicuous in loosely bound unstable nuclei. Of course the total fusion, being the sum of incomplete fusion and complete fusion, would not be affected by the DPP, which was borne out clearly in the present paper. A full discussion of this new phenomenon, the BTA, will be reported elsewhere.

For the purpose of comparison, we have followed a similar procedure for the tightly bound nucleus ${ }^{16} \mathrm{O}$ scattering from ${ }^{64} \mathrm{Zn}$. Figure 9 shows the best data fits (full lines) and the predictions from the SPOP with $\mathrm{N}_{R}=1$ and $\mathrm{N}_{i}=0.78$ (dashed lines). The results of the energy dependence of the best $\mathrm{N}_{R}$ and $\mathrm{N}_{i}$ values are shown in Figure 10. Here, the usual TA is observed: as the energy is lowered below the barrier (the threshold of nonelastic processes), $\mathrm{N}_{i}$, which is about 0.6 at energies above the barrier, suffers a significant decrease, accompained by a rather sharp increase in $\mathrm{N}_{R}$ (more attraction). This is fully consistent with the prediction of the dispersion relation. 


\section{SUMMARY AND CONCLUSIONS}

Total fusion cross sections at 16, 18, 20, 22 and $24 \mathrm{MeV}$ projectile energies were measured for the ${ }^{6} \mathrm{Li}+{ }^{64} \mathrm{Zn}$ system and at 20, 22 and $24 \mathrm{MeV}$ for the ${ }^{9} \mathrm{Be}+{ }^{64} \mathrm{Zn}$ system. In addition, reaction cross sections were also derived for some energies using the present data and previously measured data which include those obtained at energies above the barrier for the reaction ${ }^{7} \mathrm{Li}+{ }^{64} \mathrm{Zn}$. For the ${ }^{16} \mathrm{O}+{ }^{64} \mathrm{Zn}$ system, we were able to derive reaction cross sections in a wide energy range (40-68.5 MeV) using elastic scattering data previously reported.

We conclude that for the fusion of ${ }^{9} \mathrm{Be}$ with the medium mass target ${ }^{64} \mathrm{Zn}$, the $\mathrm{TF}$ and the sum of the contributions of ${ }^{9} \mathrm{Be}$ and ${ }^{8} \mathrm{Be}$ to fusion are not affected by the break-up process, at least within the experimental uncertainties. The $\alpha$-ICF cross section seems to be very small and therefore can be ignored. The EBU contribution is important only at near and sub-barrier energies. In the reactions involving projectiles ${ }^{6,7} \mathrm{Li}$ and ${ }^{6} \mathrm{He}$, with the same target, the $\sigma_{R}$ and the $\sigma_{E B U}$ are large at all energies, and both increase as the break-up threshold energy gets smaller. However, the total fusion cross-section is not affected by the break-up channel.

The energy dependence of the real and imaginary parts of the potential was studied by the double folding global São Paulo potential, and the results indicate the operation of a new type of threshold anomaly in the elastic scattering of the weakly bound nucleus ${ }^{9} \mathrm{Be}$ : The imaginary potential suffers a significant rise as the energy is lowered below the barrier, accompanied by a sharp decrease of the real potential. This favors an increase in the barrier height and should result in a decrease in sub-barrier complete fusion. This new, Breakup Threshold Anomaly (BTA), is in sharp contrast to the usual threshold anomaly (TA) seen in the energy dependence of the potential in the case of the elastic scattering of the tightly bound ${ }^{16} \mathrm{O}$. The existence of the BTA is believed to be due to the presence of the strong coupling to the break-up channel, with large cross section at energies close and below the Coulomb barrier, and therefore the imaginary potential does not decrease, in fact it increases, as the energy decreases towards the Coulomb barrier.

\section{Acknowledgements}

The authors would like to thank the CNPq, CAPES, FAPERJ, FAPESP and CONICET 
for their financial support.

[1] J.J. Kolata et al., Physical Review Letters 81, 4580 (1998).

[2] M. Trotta et al., Physical Review Letters 84, 2342 (2000).

[3] E.F. Aguillera et al. , Physical Review C 63, 061603(R) (2001).

[4] A. Yoshida et al., Physics Letters B 389, 457 (1996).

[5] C. Signorini et al., European Physical Journal A 2, 227 (1998).

[6] K. E. Rehm et al., Physical Review Letters 81, 3341 (1998).

[7] P.R.S. Gomes, J. Lubian, R.M. Anjos, Nuclear Physics A 734, 233 (2004).

[8] P.R.S. Gomes, R.M. Anjos, J. Lubian, Progress of Theoretical Physics Suplement 154, 92 (2004).

[9] P.R.S. Gomes, R.M. Anjos, J. Lubian, Brazilian Journal of Physics 34, 737 (2004).

[10] M.S. Hussein, Nuclear Physics A 531, 192 (1991).

[11] N. Takigawa, H. Sagawa, Physics Letters B 265, 23 (1991).

[12] M.S. Hussein, M.P. Pato, L. F. Canto, R. Donangelo, Physical Review C46, 377 (1992).

[13] N. Takigawa, M. Kuratani, H. Sagawa, Physical Review C47, R2470 (1993).

[14] M.S. Hussein et al., Nuclear Physics A588, 85c (1995).

[15] L.F.Canto, R. Donangelo, P. Lotti, M.S. Hussein, Physical Review C52, R2848 (1995).

[16] M.S. Hussein, L.F. Canto, R. Donangelo, Nuclear Physics A 722, 321 (2003).

[17] W.H. Z. Cardenas et al., Nuclear Physics A 703 , 633 (2002).

[18] W.H.Z. Cardenas et al., Physical Review C 68 , 054614 (2003).

[19] K. Hagino, N. Rowley, A.T. Kruppa, Computer Physics Communications 123, 143 (1999).

[20] N. Keeley, K.W. Kemper, K. Rusek, Physical Review C 65, 014601 (2002).

[21] K. Hagino, A Vitturi, C.H. Dasso, S.M. Lenzi, Physical Review C61, 037602 (2000).

[22] A. Diaz-Torres, I.J. Thompson, Physical Review C 65, 024606 (2002).

[23] A. Raabe et al., Nature 431, 823 (2004).

[24] M. Dasgupta et al., Physical Review Letters 82, 1395 (1999).

[25] M. Dasgupta et al., Physical Review C 66, 041602(R) (2002).

[26] M. Dasgupta et al., Physical Review C 70, 024606 (2004).

[27] V. Tripathi et al., Physical Review Letters 88, 172701 (2002). 
[28] C. Signorini et al., European Physical Journal A 2, 227 (1998).

[29] C. Signorini et al., European Physical Journal A 5, 7 (1999).

[30] Y. W. Wu et al., Physical Review C 68, 044605 (2003).

[31] D. J. Hinde et al., Physical Re. Letters 89, 272701 (2002).

[32] G.R. Kelly et al. , Physical Review C 63, 024601 (2001).

[33] R.J. Woolliscroft et al., Physical Review C 68, 014611 (2003).

[34] C. Signorini et al., Physical Review C 67, 044607 (2003).

[35] C. Signorini, European Physical Journal A 13, 129 (2002).

[36] V. Guimarães et al., Physical Review Letters 84, 1862 (2000).

[37] P.R.S. Gomes et al., Physics Letters B 601, 20 (2004).

[38] S.B. Moraes et al., Physical Review C61, 64608 (2000).

[39] C. Tenreiro et al., Physical Review C53, 2870 (1996).

[40] A.M.M. Maciel et al., Physical Review C59, 2103 (1999).

[41] I. Padron et al., Physical Review C 66, 044608 (2002).

[42] G.V. Martí et al., submitted to Physical Review C

[43] C. Tenreiro et al. , Proceedings Workshop on Heavy Ion Fusion: Exploring the Variety of Nuclear Properties, Padova, Italy (1994), 98, Ed. A. Stefanini.

[44] J. Lubian et al., Physical Review C 64, 027601 (2001).

[45] C. Beck et al., Physical Review C67 , 054602 (2003).

[46] A. Diaz-Torrez, I.J. Thompson, C. Beck, Physical Review C 68, 044607 (2003).

[47] A. Di Pietro et al., Europhysics Letters 64, 309 (2003).

[48] A. Di Pietro et al., Physical Review C 69, 044613 (2004).

[49] N. Keeley et al., Nuclear Physics A 571, 326 (1994).

[50] A. Pakou et al., Physics Letters B 556, 21 (2003).

[51] A. Pakou et al., Physical Review C 69, 054602 (2004).

[52] P.R.S. Gomes et al., Physical Review C 70,054605 (2004).

[53] C. Signorini et al., Physical Review C61, 061603(R) (2000).

[54] R. J. Woolliscroft et al., Physical Review C 69, 044612 (2004).

[55] L. C. Chamon et al., Physical Review C 66, 014610 (2002).

[56] M. A. G. Alvarez et al., Nuclear Physics A 723, 93 (2003).

[57] N. Austern et al., Physics Report 154, 125 (1987). 
[58] M. S. Hussein, M. P. Pato, L. F. Canto and R. Donangelo, Physical Review 47, 2398 (1993).

[59] C. A. Bertulani, L. F. Canto and M. S. Hussein, Physics Report 226, 281 (1993).

[60] C. A. Bertulani, M. S. Hussein and G. Münzenberg, "Physics of Radioactive Beams" (Nova Science, New York, 2001). 
Table 1) Fusion and reaction cross sections for the different systems measured by our groups. $\sigma_{R}$ were obtained from elastic scattering data.

(a) ${ }^{6} \mathrm{Li}$ and ${ }^{7} \mathrm{Li}+{ }^{64} \mathrm{Zn}$

\begin{tabular}{|c|c|c|c|c|}
\hline $\mathrm{E}_{\text {Lab }}(\mathrm{MeV})$ & $\sigma_{\text {fus }}\left({ }^{6} \mathrm{Li}\right)(\mathrm{mb})$ & $\sigma_{R}\left({ }^{6} \mathrm{Li}\right)(\mathrm{mb})$ & $\sigma_{\text {fus }}\left({ }^{7} \mathrm{Li}\right)(\mathrm{mb})$ & $\sigma_{R}\left({ }^{7} \mathrm{Li}\right)(\mathrm{mb})$ \\
\hline 16.0 & $114 \pm 13$ & & & \\
\hline 17.0 & & 533 & & \\
\hline 18.0 & $145 \pm 14$ & & & \\
\hline 20.0 & $332 \pm 25$ & 878 & & 854 \\
\hline 22.0 & $488 \pm 41$ & 1094 & & 1100 \\
\hline 24.0 & $634 \pm 48$ & & $656 \pm 56[41]$ & \\
\hline 28.0 & $823 \pm 59[41]$ & & $883 \pm 66[41]$ & \\
\hline 31.0 & $869 \pm 60[41]$ & & $922 \pm 64[41]$ & \\
\hline 34.0 & $984 \pm 68[41]$ & & $1002 \pm 69[41]$ & \\
\hline 37.0 & $1053 \pm 71[41]$ & & $1134 \pm 77[41]$ & \\
\hline 40.0 & $1022 \pm 65[41]$ & & $1105 \pm 75[41]$ & \\
\hline 43.0 & $1166 \pm 71[41]$ & & $1254 \pm 81[41]$ & \\
\hline
\end{tabular}

(b) ${ }^{9} \mathrm{Be}$ and ${ }^{16} \mathrm{O}+{ }^{64} \mathrm{Zn}$ 


\begin{tabular}{|c|c|c|c|c|}
\hline $\mathrm{E}_{L a b}(\mathrm{MeV})$ & $\sigma_{\text {fus }}\left({ }^{9} \mathrm{Be}\right)(\mathrm{mb})$ & $\sigma_{R}\left({ }^{9} \mathrm{Be}\right)(\mathrm{mb})$ & $\sigma_{\text {fus }}\left({ }^{16} \mathrm{O}\right)(\mathrm{mb})$ & $\sigma_{R}\left({ }^{16} \mathrm{O}\right)(\mathrm{mb})$ \\
\hline 17.0 & & $68[38]$ & & \\
\hline 19.0 & & 199 [38] & & \\
\hline 20.0 & $140 \pm 18$ & & & \\
\hline 21.0 & $358 \pm 35[38]$ & $424[38]$ & & \\
\hline 22.0 & $472 \pm 46$ & & & \\
\hline 23.0 & $570 \pm 57[38]$ & $590[\underline{38}]$ & & \\
\hline 24.0 & $747 \pm 92$ & & & \\
\hline 26.0 & $930 \pm 92[38]$ & $871[38]$ & & \\
\hline 28.0 & & 1013 [38] & & \\
\hline 29.0 & $1120 \pm 112[38]$ & & & \\
\hline 40.0 & & & $60 \pm 8[43]$ & $59.3\left(39^{*}\right)$ \\
\hline 41.0 & & & & $78.9\left(39^{*}\right)$ \\
\hline 42.5 & & & $164 \pm 17[43]$ & $191\left(39^{*}\right)$ \\
\hline 43.5 & & & & $231(39 *)$ \\
\hline 44.0 & & & & $262\left(39^{*}\right)$ \\
\hline 48.0 & & & & $542\left(39^{*}\right)$ \\
\hline 50.0 & & & $536 \pm 60[43]$ & $640\left(39^{*}\right)$ \\
\hline 52.0 & & & & $727\left(39^{*}\right)$ \\
\hline 54.0 & & & & $828\left(39^{*}\right)$ \\
\hline 56.0 & & & & $914\left(39^{*}\right)$ \\
\hline 60.0 & & & $1095 \pm 110[43]$ & \\
\hline 62.0 & & & & $1120(39 *)$ \\
\hline 64.0 & & & & $1182(39 *)$ \\
\hline 68.5 & & & $1354 \pm 162[43]$ & $1350\left(39^{*}\right)$ \\
\hline
\end{tabular}

(39*)- Reaction cross sections derived from elastic scattering data reported in Ref 39 . 


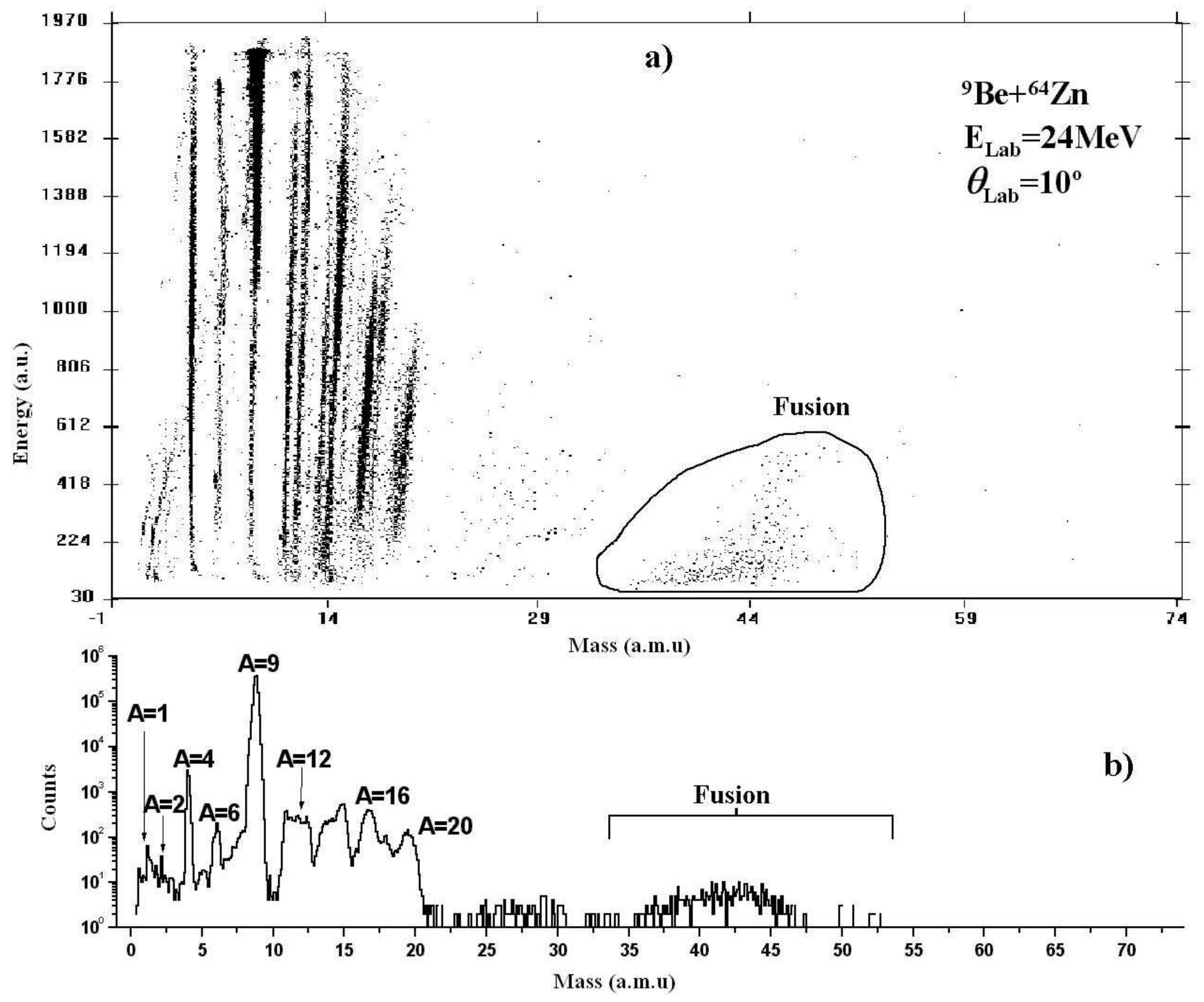




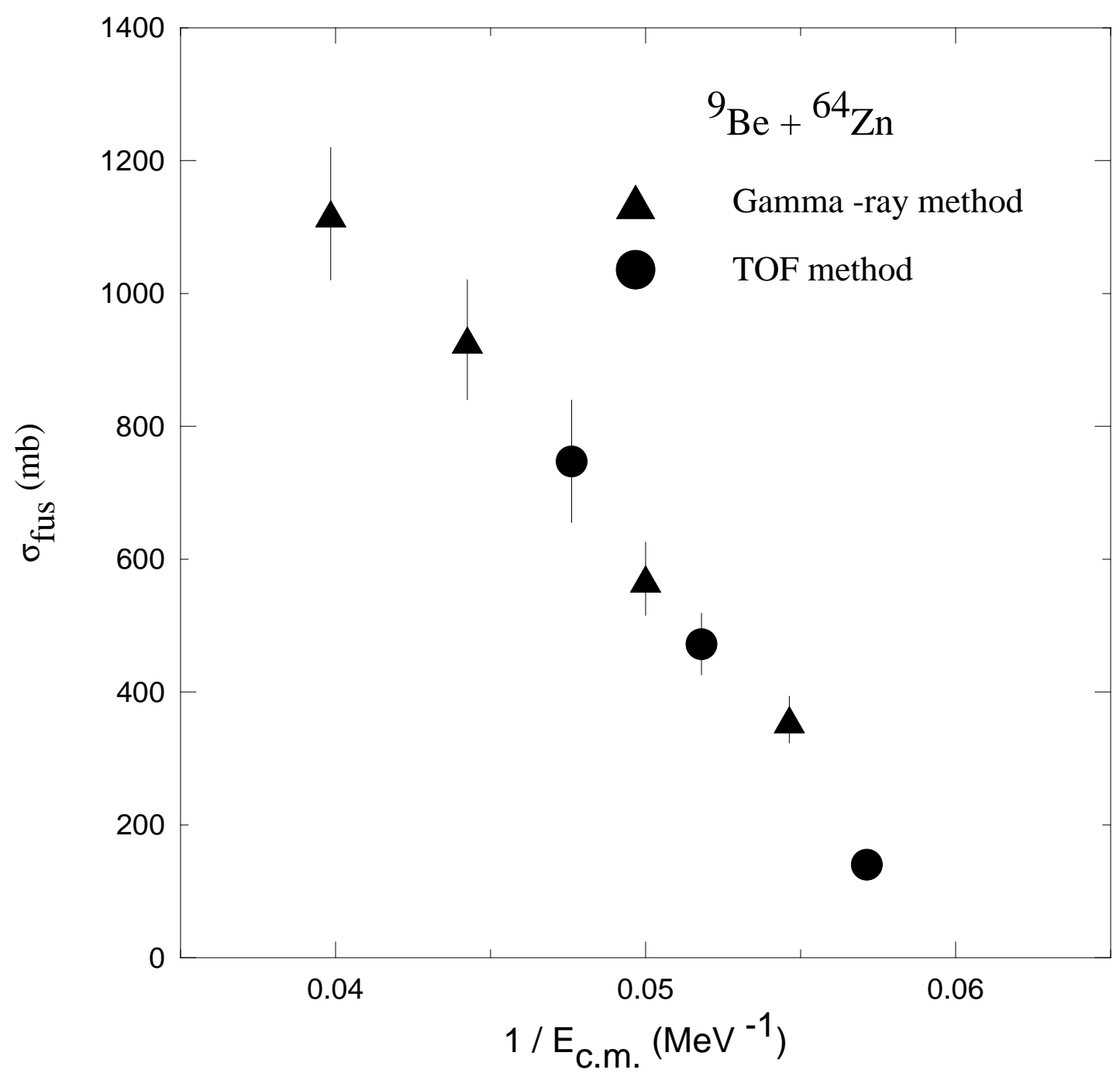




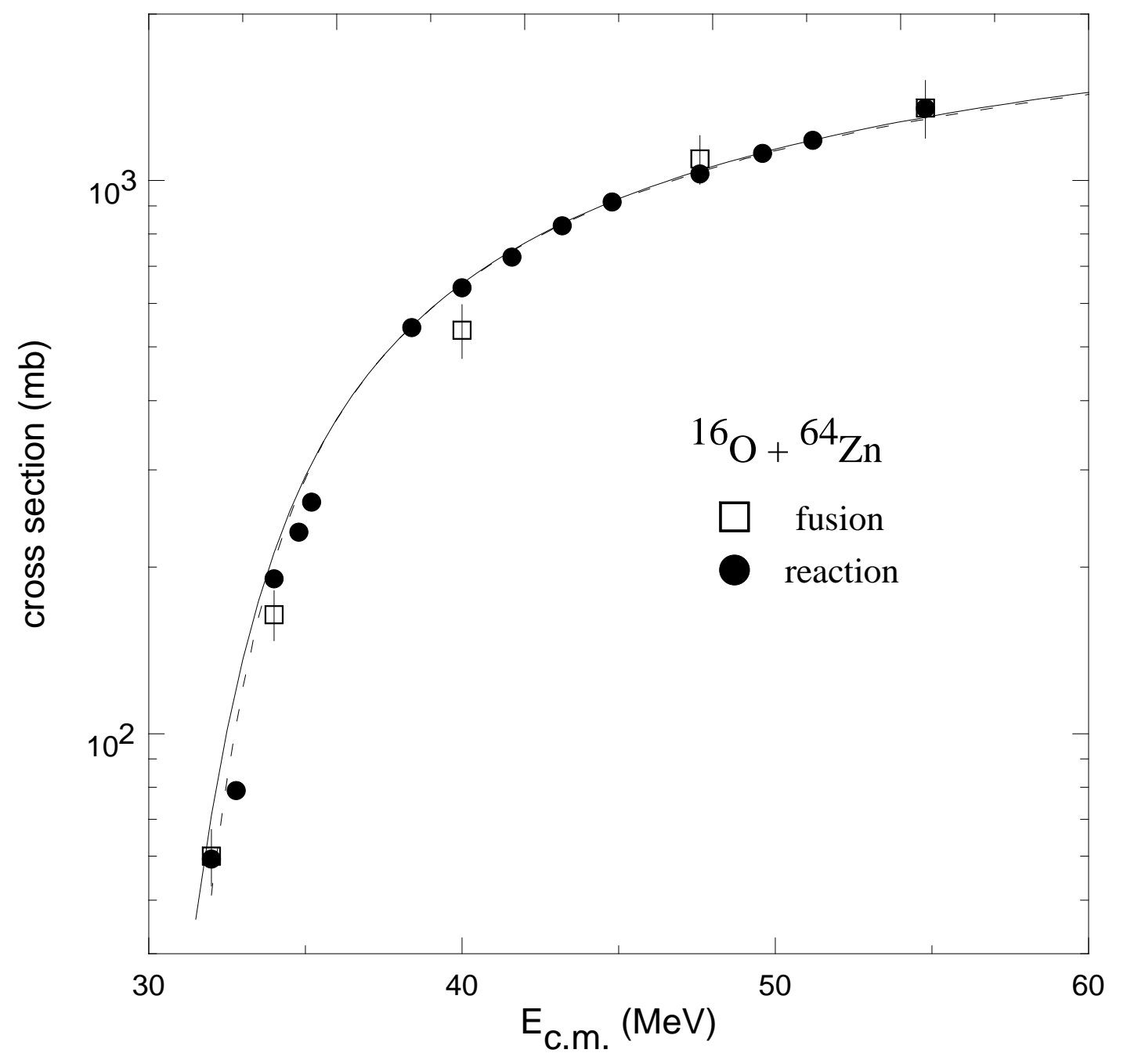




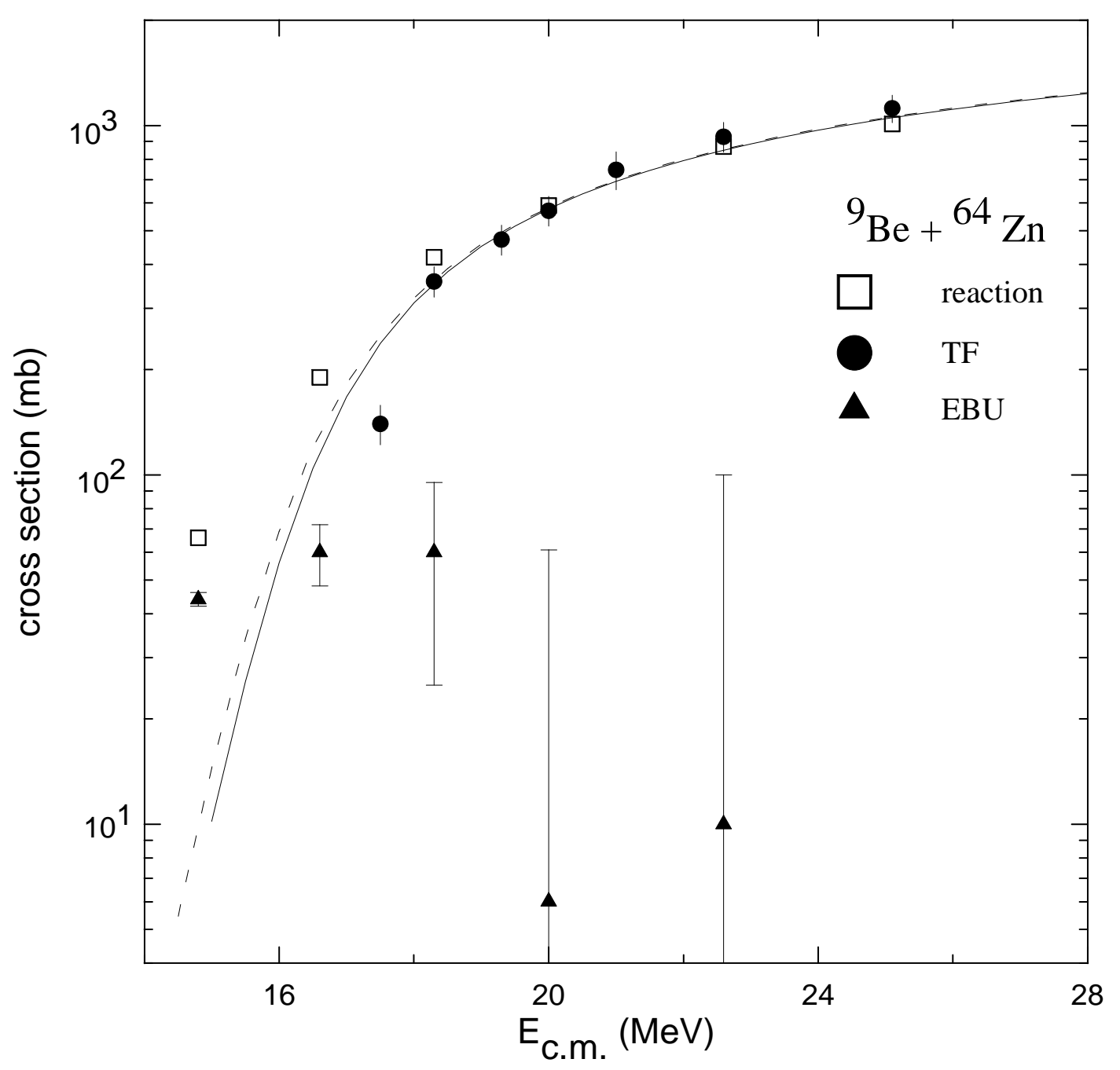




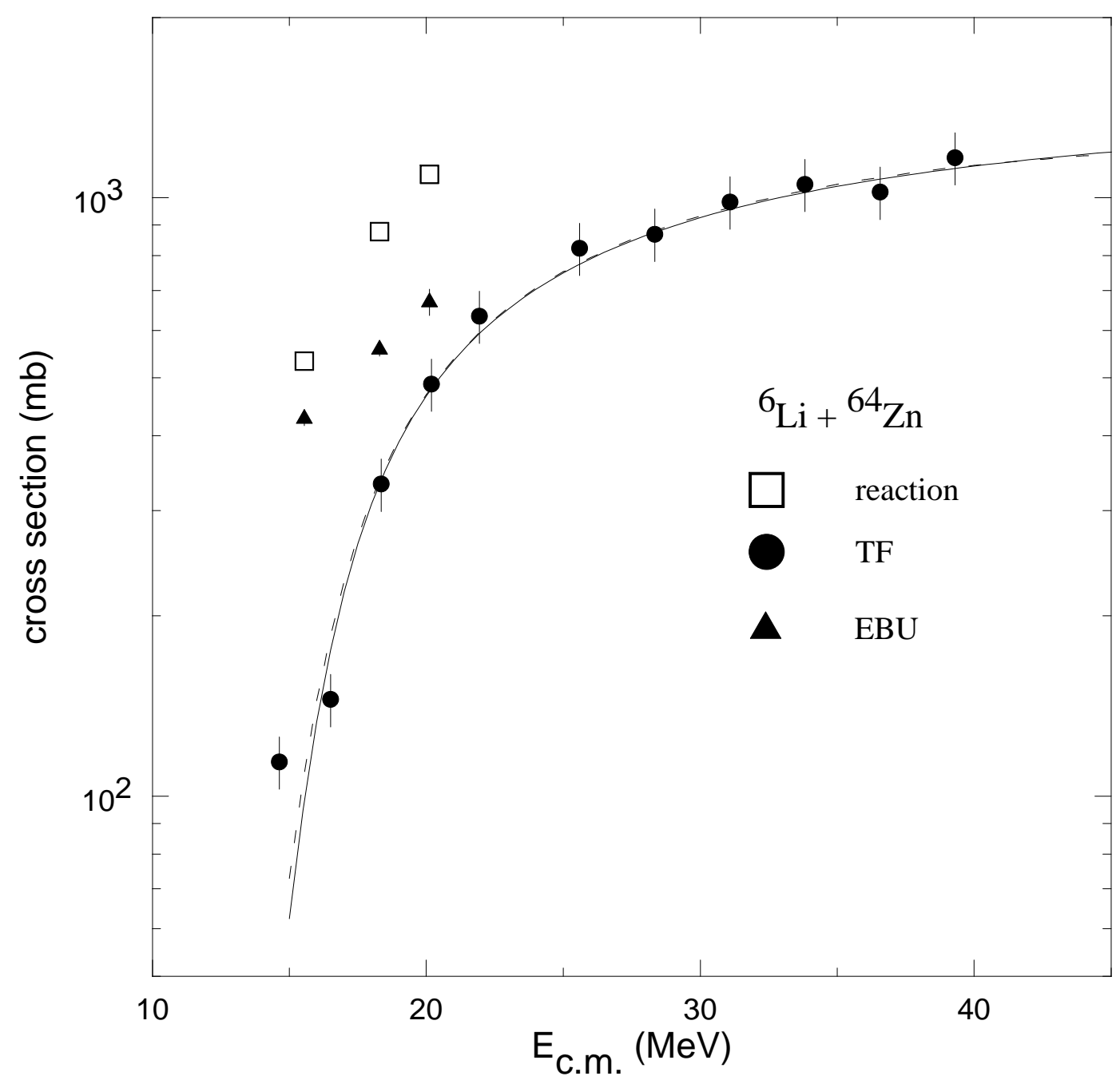




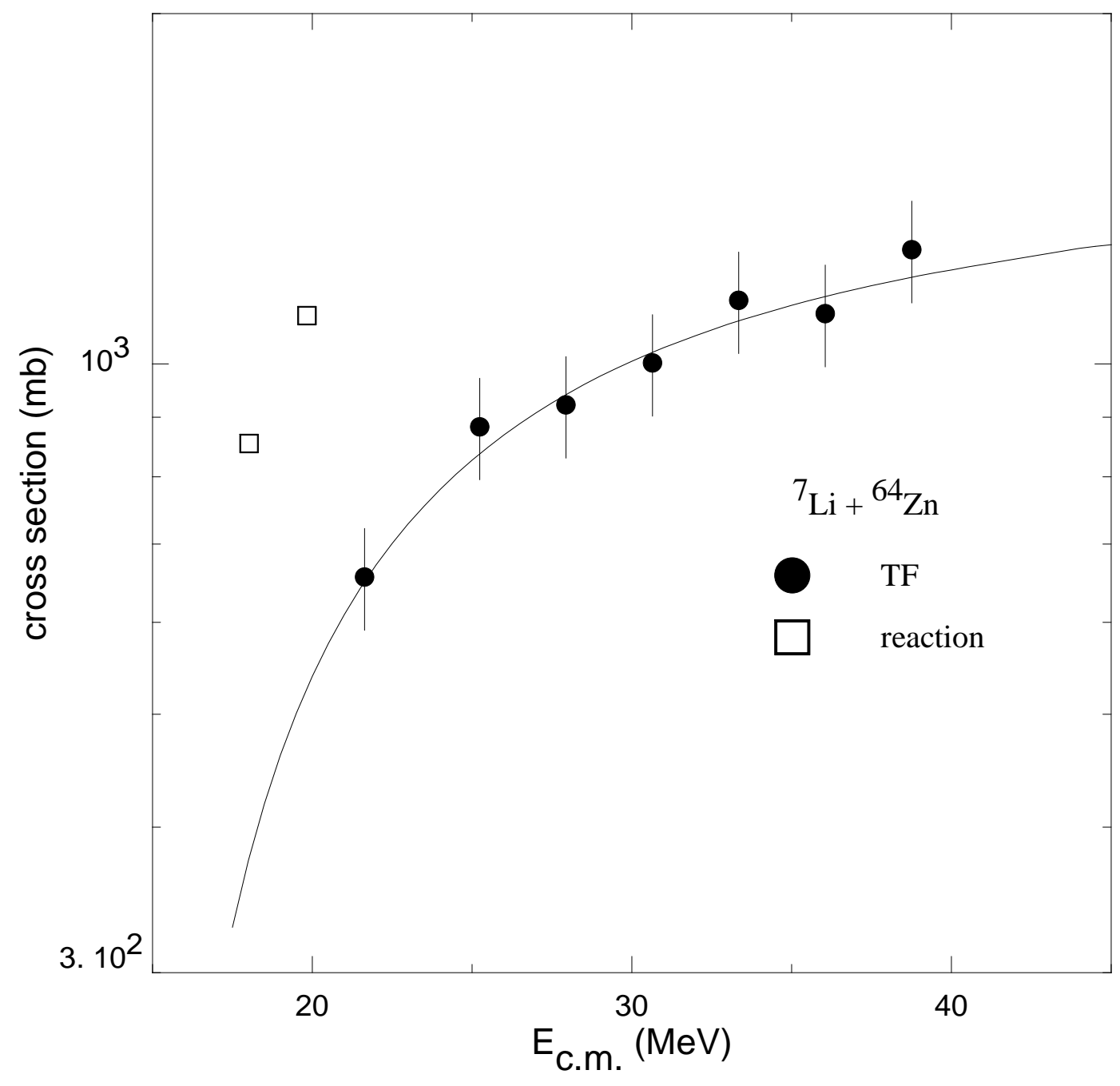



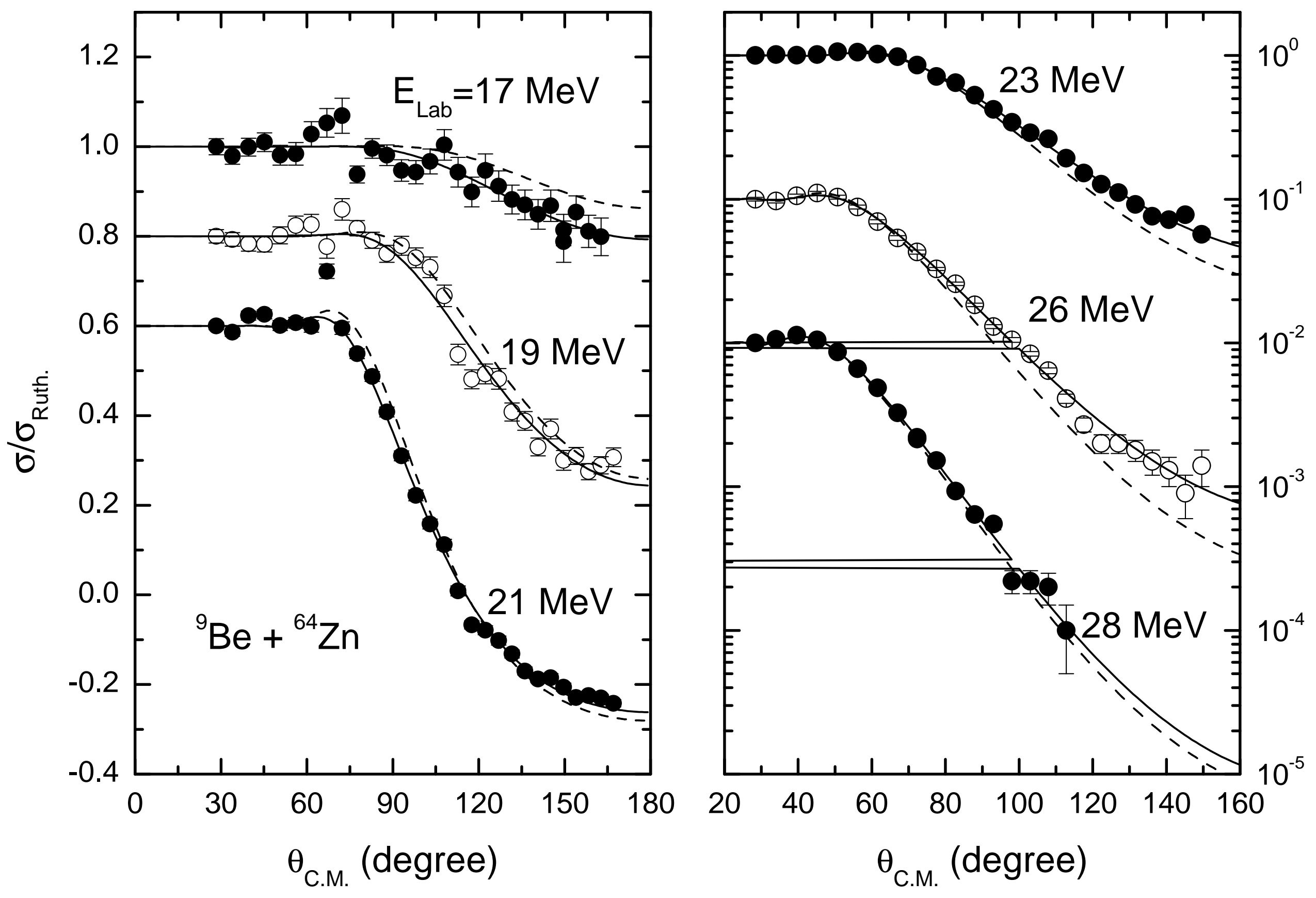


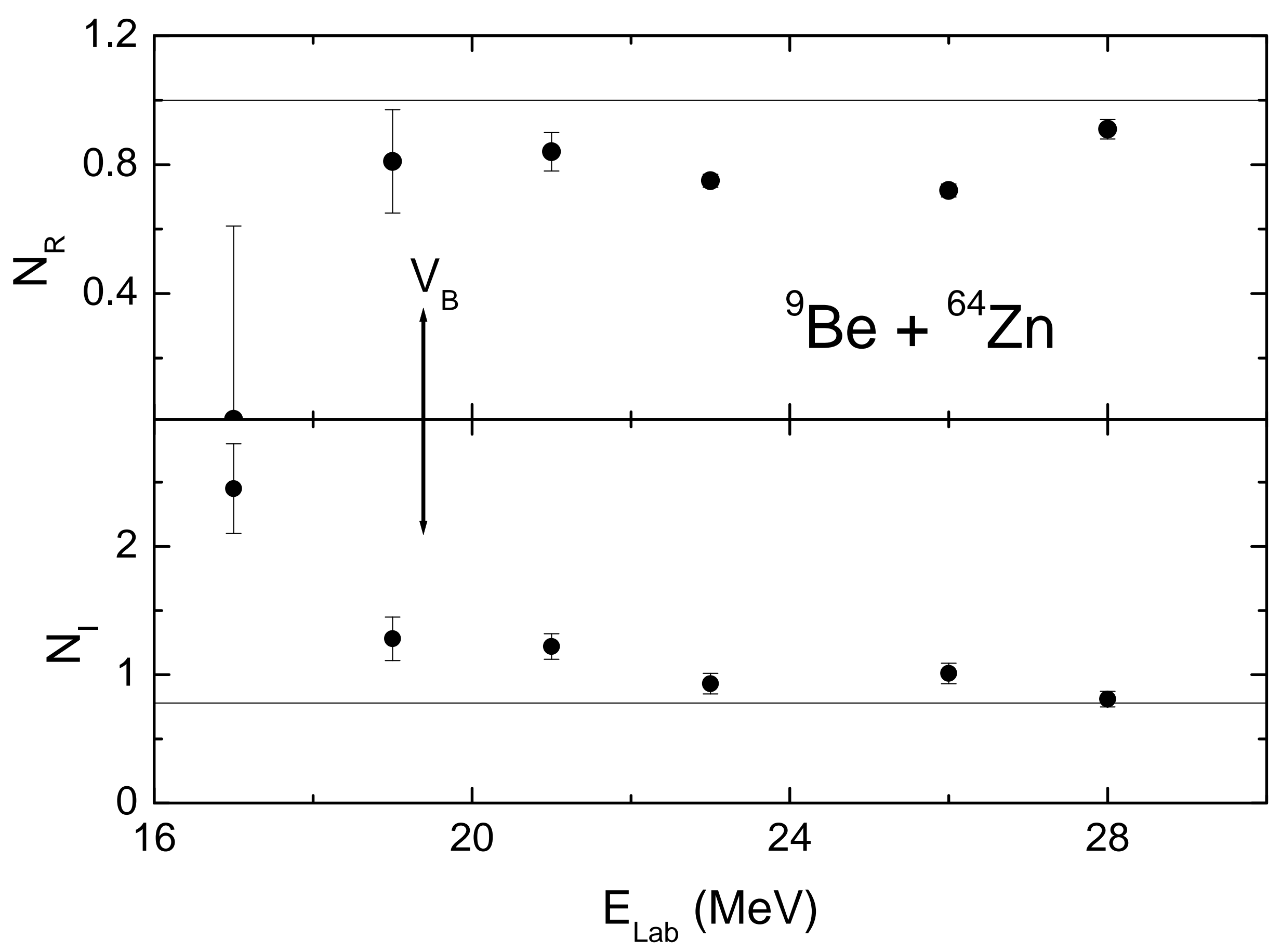




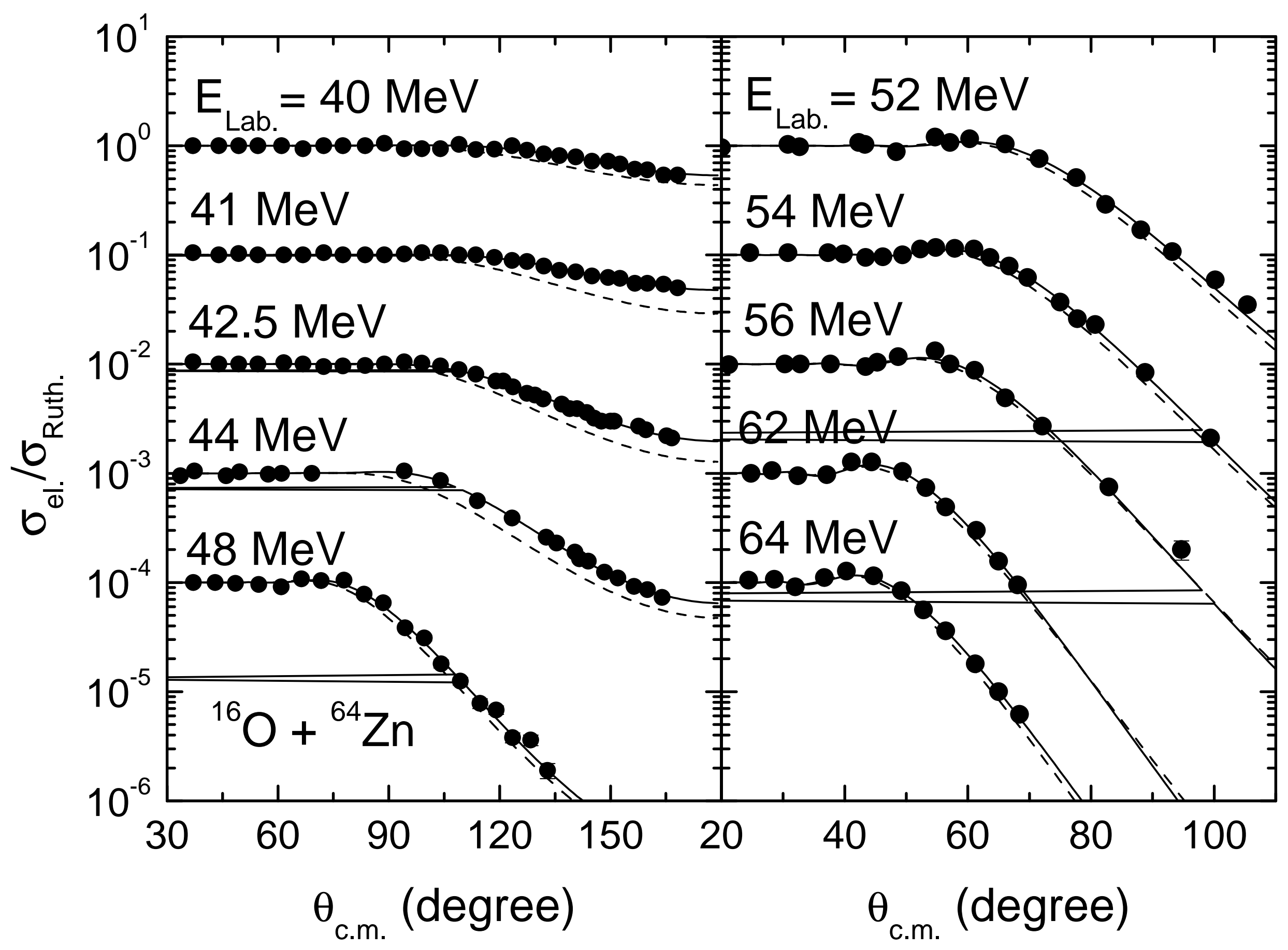




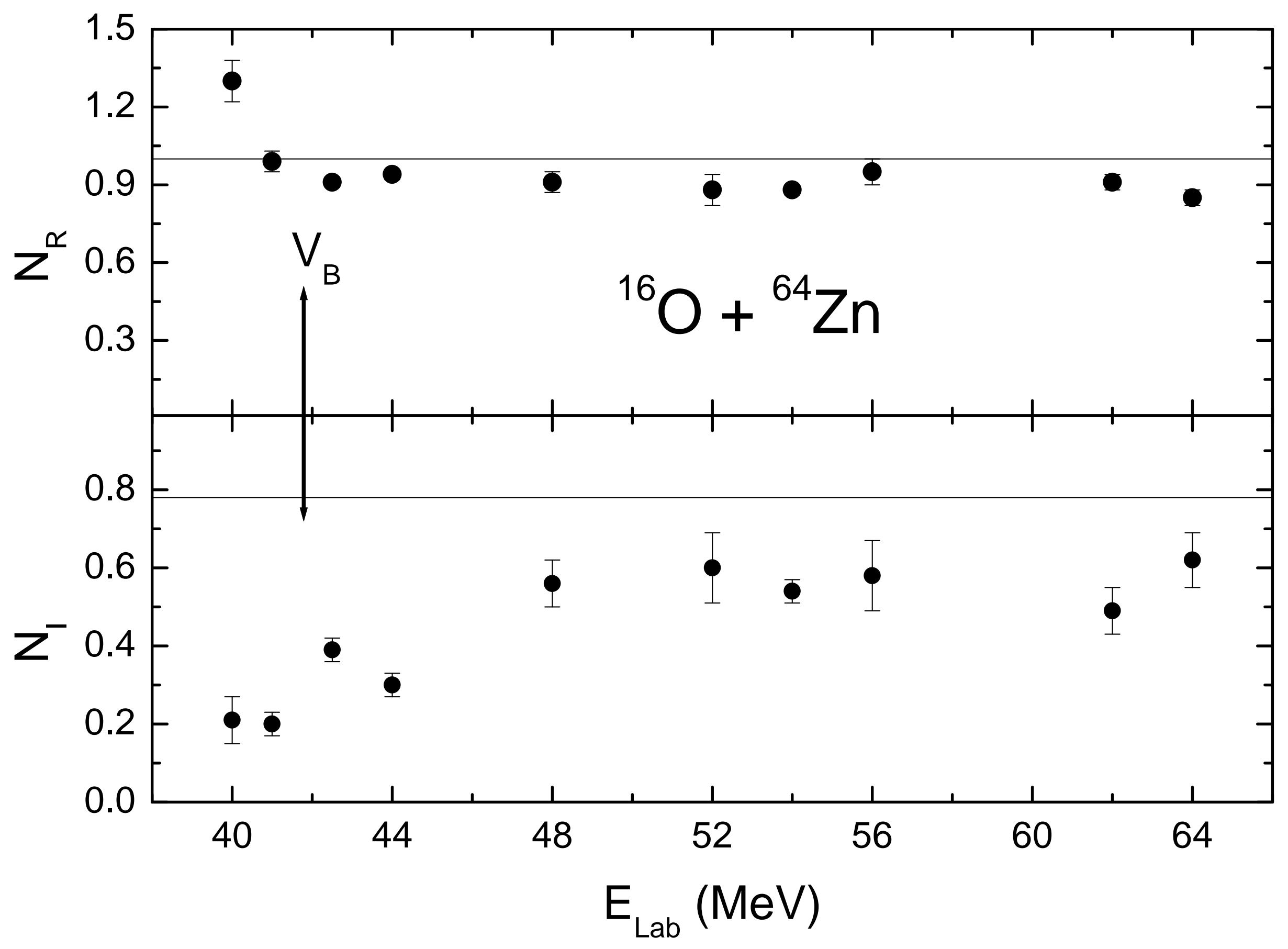

